\title{
A refined model of chronic cerebral hypoperfusion resulting in cognitive impairment and a low mortality rate in rats
}

\author{
Ahmed Mansour, MD, ${ }^{1,5}$ Kuniyasu Niizuma, MD, PhD, ${ }^{1}$ Sherif Rashad, MD, PhD, ${ }^{1}$ \\ Akira Sumiyoshi, PhD, ${ }^{2}$ Rie Ryoke, PhD, ${ }^{2}$ Hidenori Endo, MD, PhD, ${ }^{1}$ Toshiki Endo, MD, PhD, ${ }^{3}$ \\ Kenichi Sato, MD, PhD, ${ }^{4}$ Ryuta Kawashima, MD, PhD, ${ }^{2}$ and Teiji Tominaga, MD, PhD ${ }^{1}$ \\ 'Department of Neurosurgery and ${ }^{2}$ Institute of Development, Aging, and Cancer, Tohoku University Graduate School of \\ Medicine; Departments of ${ }^{3}$ Neurosurgery and ${ }^{4}$ Neuroendovascular Therapy, Kohnan Hospital, Sendai, Japan; and ${ }^{5}$ Department \\ of Neurosurgery, Menoufia University Graduate School of Medicine, Menoufia, Egypt
}

\begin{abstract}
OBJECTIVE The cognitive deficits of vascular dementia and the vasoocclusive state of moyamoya disease have often been mimicked with bilateral stenosis/occlusion of the common carotid artery (CCA) or internal carotid artery. However, the cerebral blood flow (CBF) declines abruptly in these models after ligation of the CCA, which differs from "chronic" cerebral hypoperfusion. While some modified but time-consuming techniques have used staged occlusion of both CCAs, others used microcoils for CCA stenosis, producing an adverse effect on the arterial endothelium. Thus, the authors developed a new chronic cerebral hypoperfusion $(\mathrm{CCH})$ model with cognitive impairment and a low mortality rate in rats. METHODS Male Sprague-Dawley rats were subjected to unilateral CCA occlusion and contralateral induction of CCA stenosis (modified CCA occlusion [mCCAO]) or a sham operation. Cortical regional CBF (rCBF) was measured using laser speckle flowmetry. Cognitive function was assessed using a Barnes circular maze (BCM). MRI studies were performed 4 weeks after the operation to evaluate cervical and intracranial arteries and parenchymal injury. Behavioral and histological studies were performed at 4 and 8 weeks after surgery.

RESULTS The mCCAO group revealed a gradual CBF reduction with a low mortality rate (2.3\%). White matter degeneration was evident in the corpus callosum and corpus striatum. Although the cellular density declined in the hippocampus, MRI revealed no cerebral infarctions after mCCAO. Immunohistochemistry revealed upregulated inflammatory cells and angiogenesis in the hippocampus and cerebral cortex. Results of the BCM assessment indicated significant impairment in spatial learning and memory in the mCCAO group. Although some resolution of white matter injury was observed at 8 weeks, the animals still had cognitive impairment.
\end{abstract}

CONCLUSIONS The mCCAO is a straightforward method of producing a CCH model in rats. It is associated with a low mortality rate and could potentially be used to investigate vascular disease, moyamoya disease, and $\mathrm{CCH}$. This model was verified for an extended time point of 8 weeks after surgery.

https://thejns.org/doi/abs/10.3171/2018.3.JNS172274

KEYWORDS carotid artery stenosis; cerebral blood flow; cerebral hypoperfusion; moyamoya disease; vascular dementia; white matter injury; vascular disorders

$\mathrm{T}$ HE occurrence of a stroke is attributed to a sudden shortage of cerebral blood flow (CBF) to distinct brain regions; however, a moderate but persistent reduction in the regional $\mathrm{CBF}$ ( $\mathrm{rCBF}$ ) compromises memory processes and results in the development and progression of dementia. ${ }^{5}$ Vascular dementia is characterized by cognitive impairment primarily caused by the chronic reduction of CBF. Thus, the chronic cerebral hypoperfusion $(\mathrm{CCH})$ animal model supposedly mimics vascular dementia. ${ }^{28,32,33}$

Reportedly, CCH causes white matter (WM) degen-

ABBREVIATIONS BCCAO = bilateral CCA occlusion; BCCAS = bilateral CCA stenosis; $\mathrm{BCM}=$ Barnes circular maze; $\mathrm{CBF}=$ cerebral blood flow; CCA = common carotid artery; $\mathrm{CCH}=$ chronic cerebral hypoperfusion; $\mathrm{CV}=$ cresyl violet; $\mathrm{DBP}=$ diastolic blood pressure; $\mathrm{DFn}=$ degrees of freedom in the numerator; $\mathrm{LFB}=$ luxol fast blue; $\mathrm{LSF}$ = laser speckle flowmetry; $\mathrm{mCCAO}=$ modified CCA occlusion; $\mathrm{MRA}=\mathrm{MR}$ angiography; $\mathrm{PBS}=$ phosphate-buffered saline; $\mathrm{PR}=$ pulse rate; $\mathrm{rCBF}=$ regional $\mathrm{CBF} ; \mathrm{ROI}=$ region of interest; SBP = systolic blood pressure; TOF = time of flight; VA = vertebral artery; WM = white matter.

SUBMITTED December 5, 2017. ACCEPTED March 6, 2018.

INCLUDE WHEN CITING Published online September 7, 2018; DOI: 10.3171/2018.3.JNS172274. 
eration and neuronal degradation of the hippocampal CA1 subregion ${ }^{21,23,25,29}$ and triggers oxidative stress and neuroinflammation in animal models, ${ }^{1,27}$ these diffuse brain lesions lead to spatial learning and memory impairment. ${ }^{2,3,6,16,18,32,35}$ Studies have reported various procedures of a $\mathrm{CCH}$ model, including single-stage bilateral common carotid artery (CCA) occlusion (BCCAO), unilateral CCA occlusion, staged BCCAO, and bilateral CCA stenosis (BCCAS) using microcoils. Although BCCAO induces neuronal loss and glial cell activation in the cortex and hippocampus, besides learning and memory impairments, the abrupt and severe reduction of $\mathrm{CBF}$ causes high mortality rates in rats (50\%-60\%), with significant infarctions that might not effectively represent a vascular dementia model ${ }^{4,23,32}$ Moreover, staged occlusion of both CCAs is time consuming, and the reported endothelial injury, the BCCAS model, which was later developed by placing microcoils or ameroid constrictors around the CCA to induce stenosis, was expensive. . $^{10,12,19,28,31}$

In this study, we aim to overcome the highlighted issues of the currently used $\mathrm{CCH}$ models and attempt to develop a straightforward and safe model with an improved survival rate in rats. We illustrate a modified CCA occlusion (mCCAO) model, with one-sided occlusion of the CCA and simultaneous stenosis of the contralateral CCA.

\section{Methods Animals}

All animals in this study were treated according to the code of ethics of the World Medical Association and Tohoku University guidelines based on the International Guiding Principles for Biomedical Research Involving Animals. Furthermore, animal protocols were approved by Tohoku University's administrative panel on laboratory animal care.

We randomly assigned 62 male adult Sprague-Dawley rats (age 8-10 weeks; weight 260-300 g) to 3 groups: 1) a 4-week mCCAO group $(\mathrm{n}=31), 2)$ a sham surgery group $(\mathrm{n}=19)$, and 3$)$ an extended time point (8-week) mCCAO group $(\mathrm{n}=12)$, subjected to the same protocols and procedures. All rats were maintained on a 12-hour light/dark cycle at a constant temperature of $22^{\circ} \mathrm{C} \pm 1{ }^{\circ} \mathrm{C}$ and were housed in plastic cages (4 rats/cage) with free access to food and water. A timeline summarizing the experimental protocol is presented in Fig. 1A.

\section{Modified CCA Occlusion Model}

All rats were anesthetized by a gas mixture of $70 \%$ nitrous oxide and $30 \%$ oxygen containing $1.5 \%$ isoflurane through a face mask. The rectal temperature was maintained at $37^{\circ} \mathrm{C}$ during surgery by means of a homeothermic blanket. After a midline ventral neck skin incision was made, both CCAs were carefully dissected from their sheaths and exposed. The left CCA was permanently occluded using a 6-0 silk suture, and the right CCA was gently banded with a 6-0 silk suture tied around both the artery and a blunt 29-gauge needle, which was then quickly withdrawn (Fig. 1B). In this study, this needle technique preserved portions of the blood flow, facilitating various degrees of artery stenosis by use of different gauge nee- dles. The 29-gauge needle was used because our pilot study showed that this size provided a satisfactory degree of stenosis (data not shown). Although this model induced one-sided occlusion and contralateral side stenosis in the early stage, the stenosis side was spontaneously occluded a few days later, resulting in bilateral occlusion (data not shown). Furthermore, sham-operated controls $(n=19)$ underwent dissection and exposure of the CCAs, as described above, without any carotid artery ligation. We recorded the survival rates of each group during the experiment.

\section{Physiological Parameters}

We assessed the systolic (SBP) and diastolic (DBP) blood pressure and pulse rate (PR) under anesthesia before and just after CCA occlusion and stenosis using a rat tail cuff blood pressure monitor (Softron Tokyo BP-98AL V3.02).

\section{CBF Measurement}

We used 9 rats from the mCCAO group and 6 from the sham-operated group for the $\mathrm{CBF}$ measurement. The $\mathrm{rCBF}$ of the frontal and parietal cortices after surgery was determined using laser speckle flowmetry (LSF; Omega Zone, Omegawave) as described previously, ${ }^{12}$ with some modifications. Briefly, we made a midline scalp incision while the animals were in a state of general anesthesia (1.5\% isoflurane) and thinned the skull using a microdrill until only a small translucent sheet of bone remained over the intact dura (Fig. 1C). Then we set a circular region of interest (ROI; diameter $2 \mathrm{~mm}$ ) at the point $3.5 \mathrm{~mm}$ posterior and $3 \mathrm{~mm}$ lateral to the bregma for assessment of the rCBF. The rCBF was recorded just before surgery and 1 hour and 3, 7, 14, 21, and 28 days after the surgery. Notably, the mean blood flow values of both sides were expressed as a percentage of the baseline values.

In a pilot study, the rCBF measured through a thinned skull, as described above, was compared with the open window measurement (total removal of the bone), and it showed no differences throughout the 4 weeks of the observational period (data not shown). Thus, the skullthinning method was selected as less invasive and more efficient.

\section{Magnetic Resonance Imaging}

We performed MRI on 6 mCCAO and 4 sham rats 4 weeks after the surgery using a dedicated small-animal scanner at $7 \mathrm{~T}$ (PharmaScan, Bruker BioSpin). In addition, time-of-flight (TOF) MR angiography (MRA) with a 3D fast low-angle shot method validated occlusion or stenosis of CCAs and collateral blood flow changes. Scan parameters were as follows: TE $2.5 \mathrm{msec}$, TR $15 \mathrm{msec}$, matrix $256 \times 128$, slice thickness $0.16 \mathrm{~mm}$, and scan duration 13 minutes and 32 seconds, average, for all scans. Coronal T2-weighted images were acquired to confirm the absence of cerebral infarction (TE $33 \mathrm{msec}$, TR $2000 \mathrm{msec}$, matrix $256 \times 256$, slice thickness $2 \mathrm{~mm}, 10$ slices, and scan duration of 8 minutes and 32 seconds, average).

During all in vivo assessments, rats were anesthetized by inhalation of $2.5 \%-3.0 \%$ isoflurane in a nitrous oxide/ oxygen carrier gas mixture through a nose cone. The am- 
A

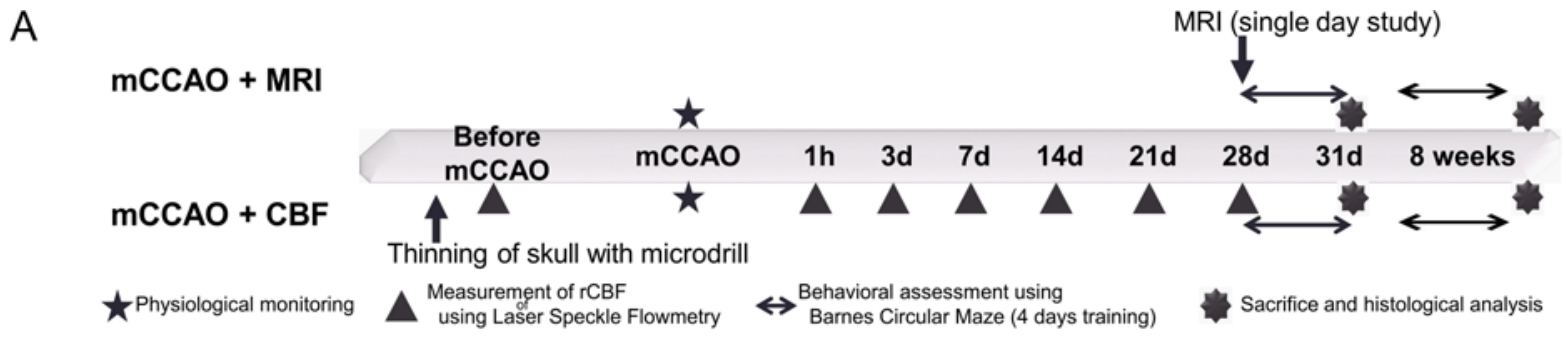

B

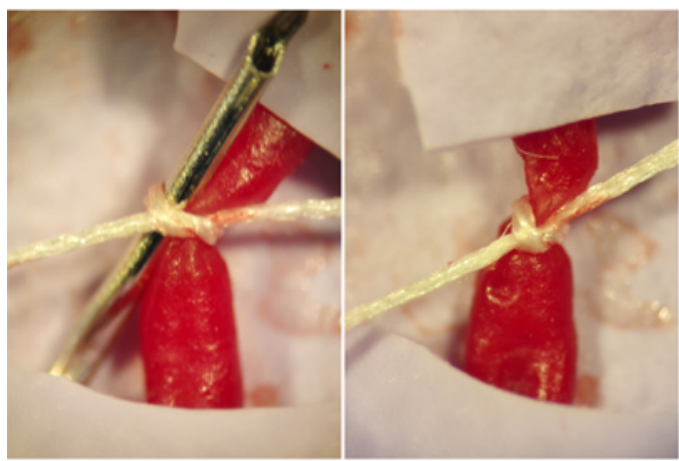

D Occlusion Stenosis

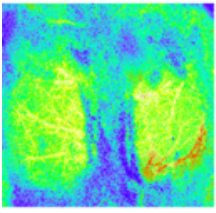

Pre

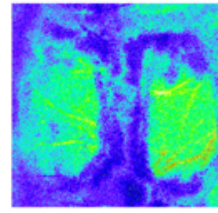

1 hour

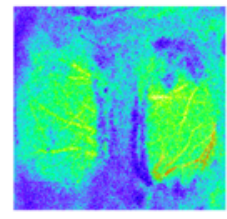

3 days
C
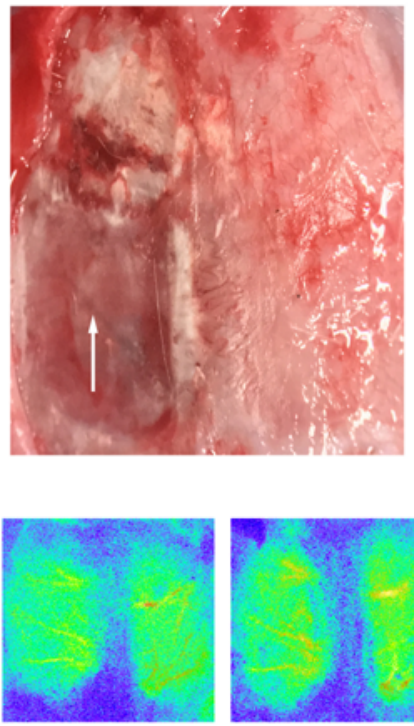

1 week

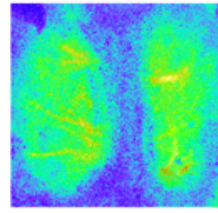

2 weeks

E

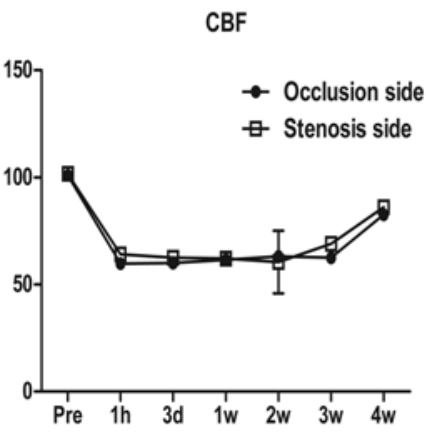

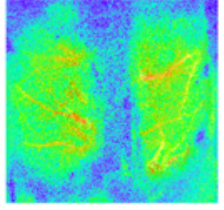

3 weeks

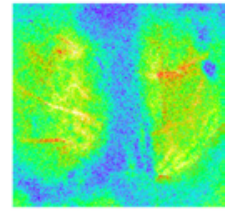

4 weeks

FIG. 1. Summary of the mCCAO model. A: Time chart showing the 2 protocols and the sequence of experiments conducted. B: Induction of severe CCA stenosis by the needle-ligature technique. $\mathbf{C}$ : The thinned skull as viewed through the surgical microscope. The left side of the skull is thinned in this image, showing a visible cortical vessel (arrow), while the right half of the skull is normal thickness. The right side would also be thinned before mean blood flow measurement. D and $\mathrm{E}$ : The temporal profile of the rCBF after mCCAO. The rCBF color map measured by LSF (D) and a graph of the mean rCBF values (from 10 animals) as percentage of baseline (E). The rCBF significantly decreased immediately after the surgery, decreased further a little more at 3 days, and then plateaued until the 2nd week. The rCBF increased again at 3 weeks, then reached a value near normal at 4 weeks after mCCAO. There was no significant difference in rCBF between the stenosis and occlusion sides (time difference, $p<0.001$; group difference, $p>0.05$ ). Error bars indicate standard deviations. "Pre" indicates baseline (before mCCAO); $d=$ days; $h=$ hours; $w=$ weeks.

bient temperature was maintained at $37^{\circ} \mathrm{C}$ with warm air, and the animals' respiration was monitored throughout the procedure. We analyzed images for quantitative assessments by NIH ImageJ software version 1.41 (https:// imagej.nih.gov/ij/). ${ }^{26}$ Vertebral artery (VA) length was measured from the plane of the intracranial entry point of the VAs to the plane of CCA bifurcation; the VA diameter was calculated as the average of 3 measurements from the intracranial segment. The proximal CCA diameter was set as the unified scale for size corrections in semiquantitative measurements; the CCA stenosis percentage was measured at the maximum stenotic segment, and then all values were analyzed as percentage of values in the sham group.

\section{Behavioral Assessment Using the Barnes Circular Maze}

At 28 days after the surgery, we assessed 9 mCCAO and 6 sham-operated rats for cognitive impairment using the Barnes circular maze (BCM). In addition, 12 rats from the extended study group were assessed 8 weeks after the surgery. The BCM is a dry land maze test for the assessment of spatial learning and memory. ${ }^{7,9,35}$ In this study we evaluated the delay in location of the escape cage (latency to escape box) as a means of assessing cognitive deficit after mCCAO.

We performed the Barnes task on "dry land," a gray acrylic platform (122 $\mathrm{cm}$ in diameter), with 16 equidistant holes around the perimeter (O'Hara \& Co.). An overhead camera assessed the rats' performance. In the 4-day training period, 2 trials were performed per day, with a maximum trial length of 300 seconds. Each trial began with the start box positioned at the center of the maze and a rat placed inside it for 30 seconds; the rat was then permitted to explore the maze freely. After reaching the target and before being returned to its home cage, the rat was left in the escape box for 30 seconds. If the rat did not enter the escape box within 300 seconds, the experimenter gently picked it up and placed it inside the target box for $30 \mathrm{sec}-$ onds before returning it to its home cage. We assessed the latency (time to reach the target box) and the number of 
wrong holes the rats stayed at and compared the results between sham animals and the 2 mCCAO groups ( 4 and 8 weeks). Furthermore, we analyzed the average speed $(\mathrm{cm} /$ sec) moved by the rats in each trial and compared the results between the groups to exclude motor dysfunction.

\section{Histological Analysis of Hippocampal and WM Injury}

Between 28 and 33 days after surgery, the animals in the 4-week mCCAO group were deeply anesthetized with isoflurane and then perfused with saline followed by $4 \%$ paraformaldehyde in 0.1-M phosphate-buffered saline (PBS; pH 7.4). The brains were removed and postfixed in the same fixative at $4^{\circ} \mathrm{C}$ overnight, then stored in $10 \%$, $20 \%$, and $30 \%$ sucrose in PBS until the tissues sank. Coronal sections (approximately $3 \mathrm{~mm}$ thick) that included the hippocampus and cortex were obtained, embedded in optimum cutting temperature (OCT) compound, frozen in liquid nitrogen, and subsequently cut into $10-\mu \mathrm{m}$ sections with a microtome-cryostat.

To evaluate hippocampal neuronal injury, we stained the sections with $1 \%$ cresyl violet $(\mathrm{CV})$. Injury to the pyramidal cell layer of the hippocampal CA1 subregion was detected under a light microscope. The numbers of neurons in the late stages of degeneration (identified by shrunken morphology and dark staining) were counted in 3 randomly selected sections containing the CA1 subregion under 20x magnification (Keyence BZ-9000; magnification comparable to $200 \times$ with standard light microscope). The results for the 21 models and 15 sham-operated animals were averaged to obtain a value for each group.

To assess WM injury, the sections were stained with luxol fast blue (LFB) followed by CV staining, as previously described..$^{15,30}$ We obtained 3 slices/rat, and assessed 3 randomly selected fields of the corpus callosum and striatum under light microscopy at the same exposure level. NIH ImageJ was used to quantify the LFB staining. The areas covered by the LFB stain were expressed as a percentage of the total area of the WM assessed.

\section{Immunohistochemistry}

After blocking of nonspecific binding sites with $10 \%$ bovine serum albumin for 2 hours at room temperature, we incubated sections overnight with the following antibodies: rabbit anti-cleaved caspase-3 (Asp175) antibody (9661; Cell Signaling Technology) to detect cell death; rabbit anti-CD34 antibody (EP373Y; ab81289; Abcam) to detect angiogenesis; anti-GFAP antibody (astrocyte; mouse mAb 3670; Cell Signaling Technology) to detect astrocytes; and rabbit anti-Iba-1 antibody (019-19741; Wako) to detect microglia. We exposed all sections to appropriate biotinylated secondary antibodies (1:200; Vector Laboratories) and visualized with $0.01 \%$ diaminobenzidine tetrahydrochloride and $0.005 \% \mathrm{H}_{2} \mathrm{O}_{2}$ in $50 \mathrm{mmol} / \mathrm{L}$ Tris- $\mathrm{HCl}$ $(\mathrm{pH}=7.6)$. Then 2 ROIs $\left(100 \mu \mathrm{m}^{2}\right)$ were randomly chosen in the middle segment of the hippocampal CA1 subregion and frontoparietal cortex of both the right (stenosis) and the left (occlusion) sides. We calculated the numbers of immunopositive cells in each ROI and averaged the results to obtain the mean density of each cell type.

\section{Statistical Analysis}

We performed comparisons among multiple groups with 1- or 2-way analysis of variance (ANOVA). Comparisons between 2 groups were performed with the Student's unpaired t-test, using GraphPad Prism version 5.03. The latency to the escape box and the number of stays at wrong holes of the BCM were assessed with a 2-way mixeddesign ANOVA with independent-measures Bonferroni posttests on groups (sham vs 4-week mCCAO; sham vs 8-week mCCAO; 4-week mCCAO vs 8-week mCCAO) and repeated measures on time using GraphPad Prism version 5.03. Data were expressed as mean $\pm \mathrm{SD}$, and $\mathrm{p}<$ 0.05 was considered statistically significant.

\section{Results}

\section{Physiological Parameters and Mortality Rate}

Comparison of data obtained in the 3 groups (the 2 mCCAO groups and the sham-operated group) showed no significant differences in the physiological parameters (SBP, $\mathrm{p}=0.4 ;$ DBP, $\mathrm{p}=0.35 ; \mathrm{PR}, \mathrm{p}=0.38$; Table 1). All 19 animals in the sham-operated group survived until euthanasia, and only 1 of $43(2.32 \%)$ rats in the 2 mCCAO groups died during the 8-week study period.

\section{Moderate Reduction and Gradual Recovery of rCBF After mCCAO}

Relative to baseline measurements, the mean blood flow values for the 2 sides were $63 \% \pm 1.6 \%$ (occlusion side) and $73 \% \pm 2.1 \%$ (stenosis side), 1 hour after surgery. Low rCBF values lasted until the 2 nd week ( 3 days, $60 \%$ $\pm 2.7 \%$ [occlusion side] and $71 \% \pm 1.8 \%$ [stenosis side]; 1 week, $60 \% \pm 3 \%$ and $73 \pm 2.5 \%$, respectively). Then the rCBF increased ( 2 weeks, $78 \% \pm 1.2 \%$ and $82 \% \pm 3.1 \% ; 3$ weeks, $83 \% \pm 1.1 \%$ and $84 \% \pm 3.4 \%$; 4 weeks, $94 \% \pm 1.2 \%$ and $96 \% \pm 1.4 \%$, respectively; time difference, $\mathrm{p}<0.001$, $F=2.5$; Fig. 1D and E). No significant difference was observed in the rCBF between occlusion and stenosis sides over different time points (group difference, $\mathrm{p}>0.05$ ).

\section{Development of Collateral Circulation After mCCAO With No Focal Cerebral Infarctions}

In the sham-operated group, 3D-TOF MRA demonstrated that VAs were relatively faint and narrow, while bilateral CCAs were clearly visualized. In contrast, in the animals subjected to mCCAO, 4 weeks after the procedure, 3D-TOF MRA showed no CCA signal on the occlusion side and a signal defect in the CCA on the stenosis

TABLE 1. Comparison of physiological parameters just before and immediately after occlusion/stenosis of CCA

\begin{tabular}{lrrl}
\hline \multicolumn{1}{c}{ Parameter } & \multicolumn{1}{c}{ Before } & \multicolumn{1}{c}{ After } & p Value \\
\hline SBP $(\mathrm{mm} \mathrm{Hg})$ & $145.8 \pm 2.9$ & $147.8 \pm 4.8$ & $0.4(F=2.76, \mathrm{DFn}=5)$ \\
\hline $\mathrm{DBP}(\mathrm{mm} \mathrm{Hg})$ & $77.7 \pm 1.8$ & $79.3 \pm 3.8$ & $0.35(F=4.65, \mathrm{DFn}=5)$ \\
\hline PR $(\mathrm{bpm})$ & $445.2 \pm 3.5$ & $447.2 \pm 4.0$ & $0.38(F=1.28, \mathrm{DFn}=5)$ \\
\hline
\end{tabular}

$\mathrm{bpm}=$ beats per minute.

Data are presented as mean $\pm S D$ and were measured using a rat tail cuff monitor while the rat was in a state of general anesthesia. 
A

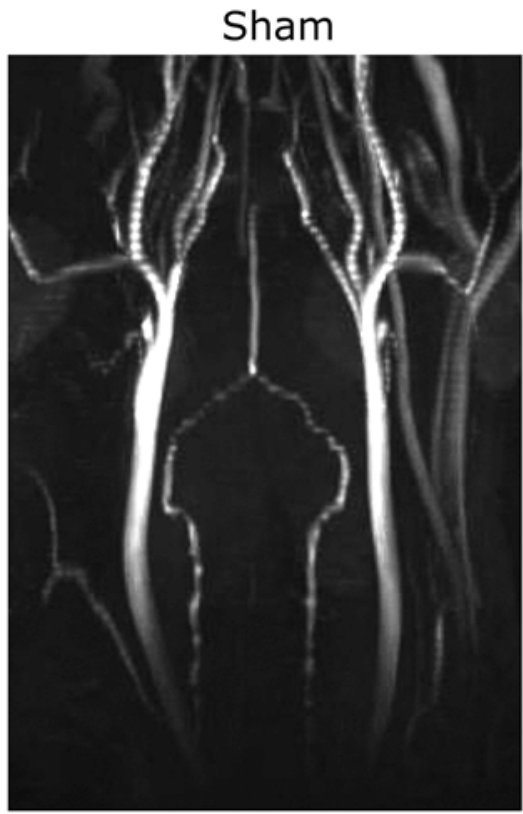

B

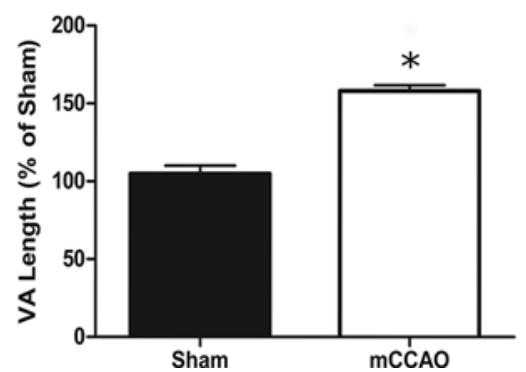

mCCAO
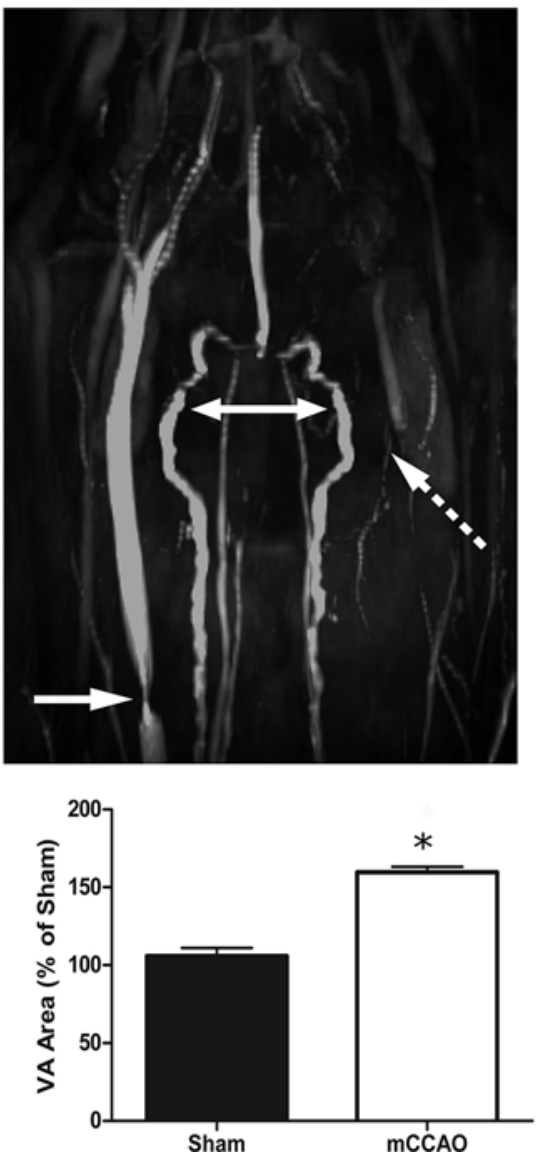

C
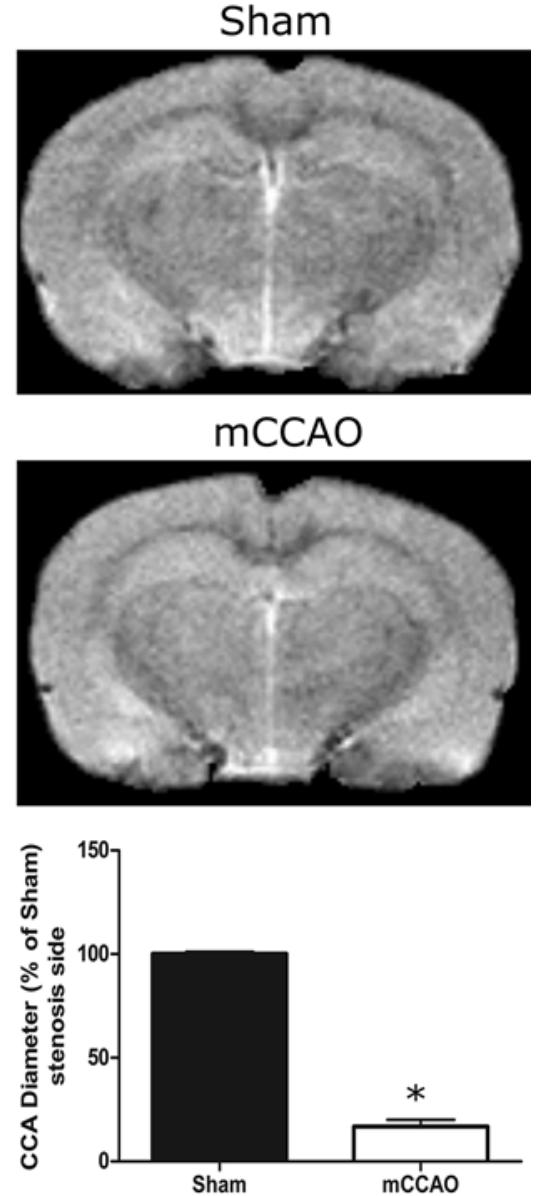

FIG. 2. Imaging findings 4 weeks after mCCAO. A: Maximum intensity projection of the 3D-TOF MRA. In sham-operated rats, the bilateral CCAs are clearly visible, whereas the VAs are faint and narrow. In contrast, in the mCCAO rat, the CCA signal at the occlusion side is not visualized (dotted arrow), and the CCA on the stenosis side shows a filling defect (arrow); the VAs (doubleheaded arrow) have thickened walls and increased diameter and tortuosity in the mCCAO group compared to shams. B: Quantification revealed a significant CCA stenosis $(83.2 \% \pm 3.2 \%, p=0.004)$; the VA length and area were increased in mCCAO rats 4 weeks after the surgery (analyzed as percentage of the values obtained in the sham group; $p=0.013$ and $p=0.009$, respectively). The graphs show means of 4 rats for the sham group and 6 rats for the mCCAO group at 4 weeks; error bars indicate standard deviations. ${ }^{*} p<0.05$. C: Coronal T2-weighted images obtained 4 weeks after surgery. No brain infarction was seen in the mCCAO or sham-operated rats.

side, while VAs showed thickened walls and increased diameter and tortuosity in the mCCAO group compared to the sham-operated controls, suggesting well-developed collateral circulations toward the anterior circulation (Fig. 2A). Data quantification demonstrated significant CCA stenosis $(83.2 \% \pm 3.2 \%, \mathrm{p}=0.004)$ in the animals that had been subjected to mCCAO. Also, 4 weeks after the surgery the VA length and area were increased as percentages of the values in the sham group (VA length, $158.1 \% \pm 3.5 \%$, $\mathrm{p}=0.013$; VA area, $159.8 \% \pm 3.5 \%, \mathrm{p}=0.009$; Fig. 2B).

T2-weighted MRI revealed no high-intensity lesion in the parenchyma, demonstrating no cerebral infarction after mCCAO (Fig. 2C). These results revealed that mCCAO caused moderate ischemia without cerebral infarction, with the development of collateral circulation through the vertebral and basilar arteries.

\section{Cognitive Dysfunction After mCCAO}

During training days 1 and 4, example paths were dem- onstrated by representative tracking plots, and the paths (complexity of the paths) and attitude (amount of hesitation) of rats presented with the maze 4 and 8 weeks after mCCAO were compared to those of the sham-operated rats (Fig. 3A). The latency of escape to goal in mCCAO rats was significantly longer than that of the controls in the 4-day training (day 1: sham, $181.8 \pm 83.1$ seconds; 4-week mCCAO, $262.3 \pm 48.7$ seconds, day $4:$ sham, $15.3 \pm 4.5$ seconds; 4-week mCCAO, $150.6 \pm 91.7$ seconds; group difference, $\mathrm{p}<0.01$; 8-week mCCAO, day 1: $275.8 \pm 57.7$ seconds, day 4: $172.5 \pm 66.6$ seconds; group difference, $\mathrm{p}$ $<0.001, F=16.97$, degrees of freedom in the numerator $[\mathrm{DFn}]=2,24$; time difference, $\mathrm{p}<0.01, F=44.3$, DFn $=$ 3,72 ; Fig. 3B). The latency of 8-week mCCAO rats was significantly longer than that of the 4-week mCCAO rats on days 2 and 3 (day 2: 4-week mCCAO, $192.1 \pm 66.0$ seconds; 8-week mCCAO, $265.7 \pm 62.5$ seconds; $\mathrm{p}<0.05$; day 3: 4-week mCCAO, $141.6 \pm 94.1$ seconds; 8-week mCCAO, $239.2 \pm 89.9$; p < 0.05; Fig. 3B), indicating more 
A

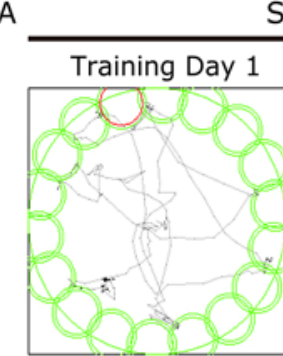

B

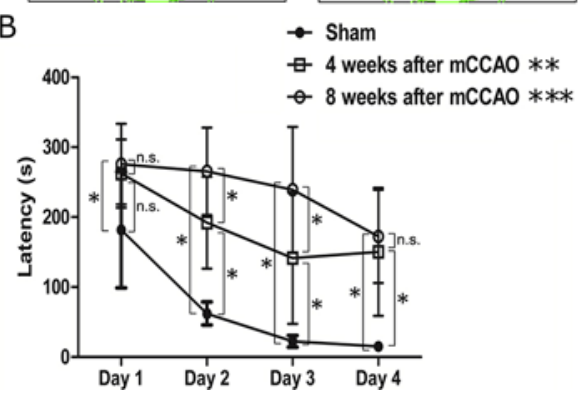

4 weeks after mCCAO

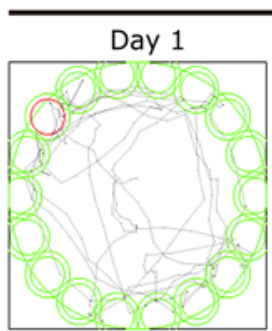

C

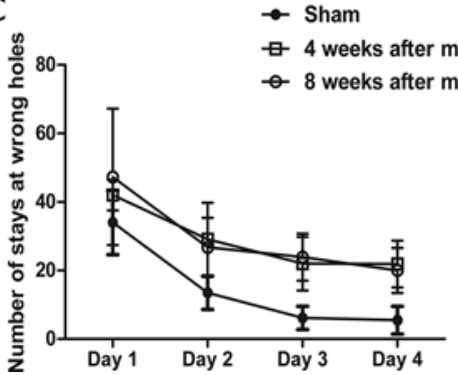

8 weeks after mCCAO

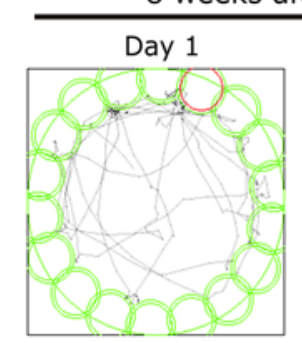

D

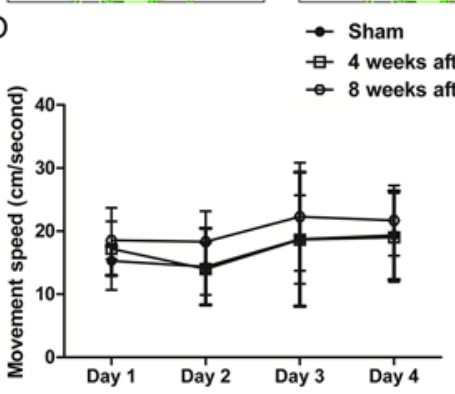

Day 4

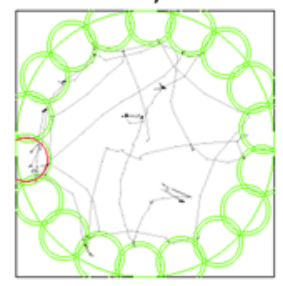

$\rightarrow$ Sham

廿 4 weeks after mCCAO mCCAO

FIG. 3. Cognitive dysfunction after mCCAO evaluated using the BCM. A: Representative diagrams of paths recorded during animal trials to detect the target box. In each set of 2 images the day 1 and day 4 diagrams show the path taken by a single rat. Comparing the paths from the 1st and 4th days of training on the BCM demonstrates a more complex and longer path to reach the target in the 4- and 8-week mCCAO groups compared to the path of the rat from the sham groups. B: Graph of time latency to find the target box revealing that the latency was significantly longer for both operation and time at all training days in the mCCAO group ( $\left.{ }^{*} p<0.05\right)$. Analysis of multiple comparisons with Bonferroni correction revealed that the latency of the 8-week mCCAO rats was significantly longer than that of sham-operated rats on day $1\left({ }^{*} p<0.05\right)$, the latency of 8-week mCCAO and 4-week mCCAO rats was significantly longer than that of sham-operated rats on day $2\left({ }^{*} p<0.05\right.$ each), the latency of 8-week mCCAO rats was significantly longer than that of 4 -week mCCAO rats on day $2\left({ }^{*} \mathrm{p}<0.05\right)$, the latency of 8-week mCCAO and 4-week mCCAO rats was significantly longer than that of sham-operated rats on day $3\left({ }^{*} p<0.05\right.$ each), the latency of 8-week mCCAO rats was significantly longer than that of 4-week mCCAO rats on day $3\left({ }^{*} p<0.05\right)$, and the latency of 8-week mCCAO and 4-week mCCAO rats was significantly longer than that of sham-operated rats on day $4\left({ }^{*} p<0.05\right.$ each). n.s. $=$ not statistically significant; $s=$ seconds. C: The number of times that rats stayed at wrong holes, demonstrating a significantly increased number for both operation and time at all training days in $\mathrm{mCCAO}$ rats $\left({ }^{*} p<0.05\right)$. D: Average movement speed. There was no significant difference between $\mathrm{mCCAO}$ and sham rats, indicating exclusive spatial learning and memory impairment. The graphs show the means of 6 rats for the sham group, 9 rats for the mCCAO group at 4 weeks, and 8 rats for the mCCAO group at 8 weeks; error bars indicate standard deviations. Figure is available in color online only.

impaired performance of 8-week mCCAO rats for spatial learning and using different search strategies. In addition, the number of times rats stayed at wrong holes increased at all days of training for 4-week and 8-week mCCAO rats compared independently to sham animals (day 1: sham, $34 \pm 9.3$ holes; 4 -week mCCAO, $42.2 \pm 4.6$; day 4: sham, $5.5 \pm 4.1$ holes; 4 -week mCCAO, $21.3 \pm 6.8$; group difference, $\mathrm{p}<0.01 ; 8$-week mCCAO, day 1: $47.2 \pm 19.8$, day 4 : $20.1 \pm 6.5$; group difference, $\mathrm{p}<0.05, F=17.21, \mathrm{DFn}=2$, 24; time difference, $\mathrm{p}<0.01, F=39.7, \mathrm{DFn}=3,72$; Fig. $3 \mathrm{C})$. The assessment of the average movement speed per rat in each trial session revealed no significant difference between 4-week mCCAO rats, 8-week mCCAO rats, and sham-operated rats (Fig. 3D), signifying spatial learning disabilities in mCCAO rats rather than motor dysfunction or inactive rats. Compared to animals in the sham group, both 4-week and 8-week mCCAO rats exhibited a significantly longer latency to detect the escape box and had significantly more stays at wrong holes. Moreover, latency to detect the escape box was longer on days 2 and 3 in the 8-week rats compared to 4-week rats, suggesting that mCCAO induced cognitive impairment, and it may be progressively deteriorated until 8 weeks after the surgery.

\section{White Matter Injury After mCCAO}

The myelin density of the corpus callosum and striatum was significantly decreased 1 week after mCCAO. At 4 weeks, the myelin density increased compared to the 1-week time point, but was significantly lower than that of the sham-operated rats (corpus callosum: sham, $8.2 \mu \mathrm{m}^{2} ; 1$ week, $5.4 \mu \mathrm{m}^{2}$; 4 weeks, $5.9 \mu \mathrm{m}^{2} ; \mathrm{p}=0.002, F=1.7$; striatum: sham, $17.4 \mu \mathrm{m}^{2} ; 1$ week, $8.4 \mu \mathrm{m}^{2}$; 4 weeks, $13.1 \mu \mathrm{m}^{2}$; $\mathrm{p}=0.001, F=2.1)$. Eight weeks after $\mathrm{mCCAO}$, the myelin density of the corpus callosum and striatum reversed toward sham values (corpus callosum: $7.6 \pm 0.4 \mu \mathrm{m}^{2} ; \mathrm{p}=$ $0.006, F=0.5$; striatum: $17.3 \pm 0.3 \mu \mathrm{m}^{2} ; \mathrm{p}=0.09, F=1.9$; Fig. 4A and B).

\section{Hippocampal Neuronal Injury After mCCAO}

The cellular density of the hippocampal CA1 subregion as identified with $\mathrm{CV}$ staining decreased in the 4-week and 8-week mCCAO rats compared to the shams (sham, $1.3 \pm 0.2$ per $100 \mu \mathrm{m}^{2} ; 4$-week mCCAO, $11.5 \pm 0.4$ per 100 $\mu \mathrm{m}^{2} ; \mathrm{p}<0.001, F=3.5 ; 8$-week mCCAO, $8.5 \pm 1.3$ per 100 $\mu \mathrm{m}^{2} ; \mathrm{p}<0.001, F=1.8$; Fig. $\left.5 \mathrm{~A}\right)$. In addition, immunohistochemistry for cleaved caspase- 3 revealed an increase in cleaved caspase-3-positive cells in the hippocampal CA1 


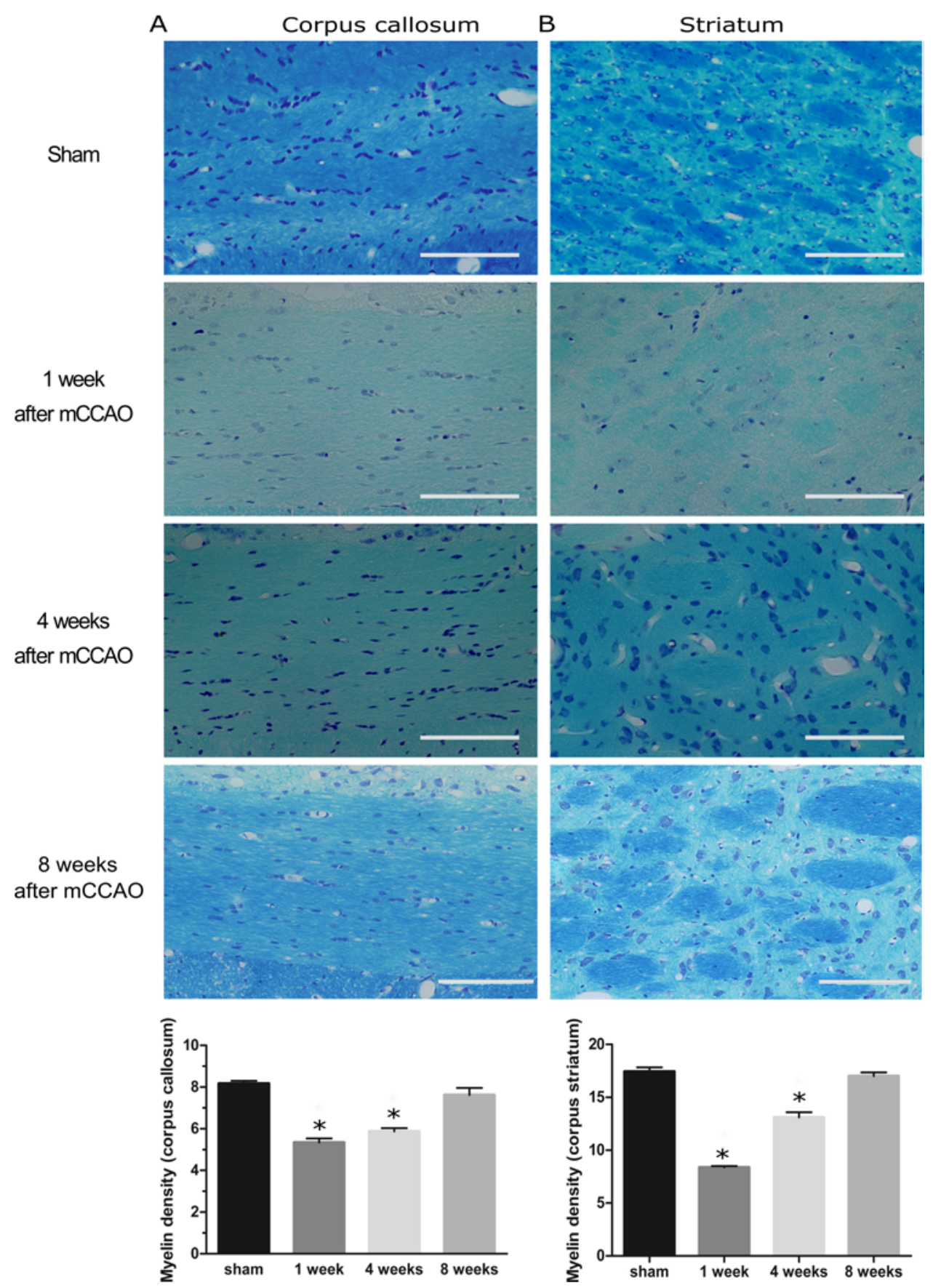

FIG. 4. White matter degeneration after mCCAO. LFB + CV staining of the mid-corpus callosum (A) and striatum (B) 1,4 , and 8 weeks after mCCAO demonstrates significantly less LFB staining density in the mCCAO group at 1 week than in the sham group $\left({ }^{*} p=0.002\right)$. Four weeks after $m C C A O$, the LFB staining density tends to increase compared to the 1-week time point but is still low compared to the value in the sham group ( ${ }^{*} p=0.001$ ). Eight weeks after $m C C A O$, the LFB staining density tends to reverse to sham group values $(p=0.09$ ). Photomicrographs, original magnification $200 x ;$ bar $=200 u m$. The graph shows the means of 6 rats for the sham group, 6 rats for the mCCAO group at 4 weeks, and 8 rats for the mCCAO group at 8 weeks; error bars indicate standard deviations.

subregion 4 and 8 weeks after mCCAO (sham, $2.5 \% \pm$ $1.6 \%$; 4-week mCCAO, $29.5 \% \pm 1.2 \% ; \mathrm{p}<0.001, F=1.1$; 8 -week mCCAO, $23 \% \pm 0.9 \%$; $<0.001, F=2.7$; Fig. 5B).

\section{Glial Activation After mCCAO}

Both GFAP and Iba-1 were increased in the hippocam- pal CA1 subregion and cerebral cortex in mCCAO rats. Compared to findings in the sham group, 4 and 8 weeks after mCCAO, the density of GFAP-positive cells (CA1: sham, $1.6 \pm 0.1$ per $100 \mu \mathrm{m}^{2}$; 4-week mCCAO, $8.9 \pm 0.2$ per $100 \mu \mathrm{m}^{2} ; \mathrm{p}=0.001, F=2.1 ; 8$-week mCCAO, $5.9 \pm 1.4$ per $100 \mu \mathrm{m}^{2} ; \mathrm{p}=0.001, F=4.7$; cortex: sham, $1.8 \pm 0.2$ 
A

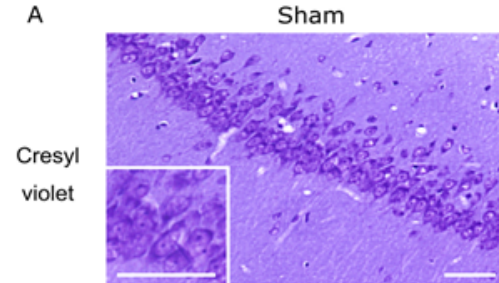

B

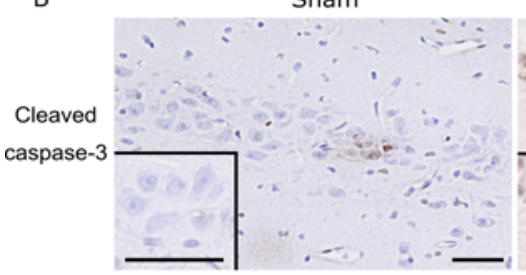

4 weeks

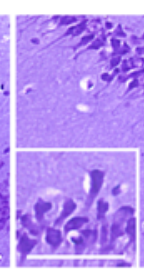

8 weeks

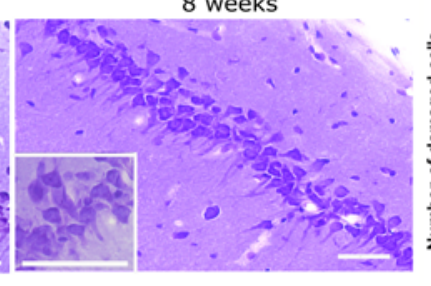

8 weeks

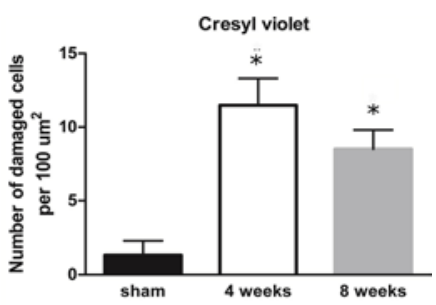

Cleaved caspace-3
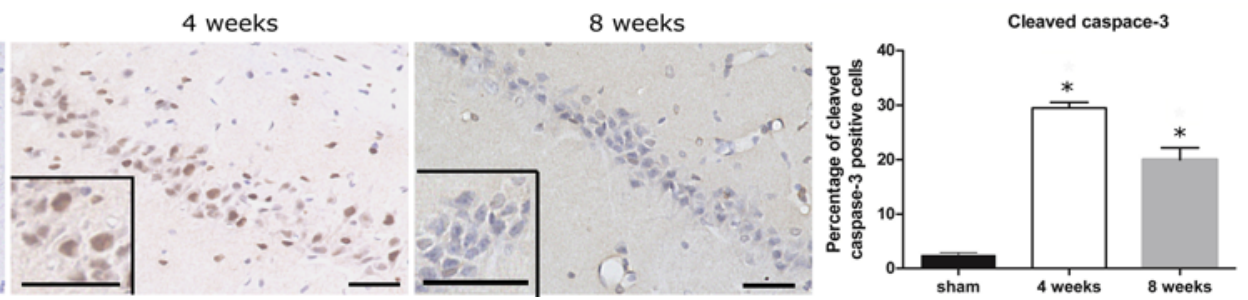

FIG. 5. Morphological changes in the neuronal cells in the hippocampal CA1 subregion. A: CV staining of the hippocampal CA1 area. The number of damaged cells (pyknotic nuclei and dark, shrunken cytoplasm) was significantly increased in the hippocampal CA1 subregion in the mCCAO group compared to the sham group $\left({ }^{*} p<0.001\right)$. Bar $=50 \mu \mathrm{m}$. B: Immunohistochemistry of cleaved caspase-3 at 4 and 8 weeks after mCCAO; the percentage of cleaved caspase-3-positive cells was increased in the hippocampal CA1 subregion 4 and 8 weeks after $\mathrm{mCCAO}\left({ }^{*} \mathrm{p}<0.001\right)$. Photomicrographs, original magnification $200 x ;$ bar $=50 \mu \mathrm{m}$. The graph shows the means of 15 rats for the sham group, 21 rats for the mCCAO group at 4 weeks, and 8 rats for the mCCAO at 8 weeks; error bars indicate standard deviations.

per $100 \mu \mathrm{m}^{2}$; 4-week mCCAO, $6.7 \pm 0.4$ per $100 \mu \mathrm{m}^{2}$; p $=0.001, F=5.8 ; 8$-week mCCAO, $6.5 \pm 1.6$ per $100 \mu \mathrm{m}^{2}$; $\mathrm{p}=0.001, F=6.4$; Fig. $6 \mathrm{~A}$ ) and Iba-1-positive cells (CA1: sham, $1.4 \pm 0.1$ per $100 \mu \mathrm{m}^{2} ; 4$-week mCCAO, $4.6 \pm 0.3$ per $100 \mu \mathrm{m}^{2} ; \mathrm{p}=0.002, F=3.8 ; 8$-week mCCAO, $6.1 \pm$ 0.8 per $100 \mu^{2} ; \mathrm{p}=0.001, F=1.4$; cortex: sham, $1.7 \pm 0.2$ per $100 \mu \mathrm{m}^{2} ; 4$-week mCCAO, $5.7 \pm 0.2$ per $100 \mu \mathrm{m}^{2} ; \mathrm{p}=$ $0.002, F=1.6$; 8 -week mCCAO, $4.5 \pm 0.9$ per $100 \mu \mathrm{m}^{2}$; $\mathrm{p}=0.002, F=3.3$; Fig. $6 \mathrm{~B}$ ) was significantly increased in the hippocampal CA1 subregion and the cerebral cortex. Reactive astrocyte and microglia were activated after $\mathrm{mCCAO}$ and contributed to neuroinflammation.

\section{Angiogenesis After mCCAO}

After 4 and 8 weeks of mCCAO, CD34-positive cells (positive microvessels) were upregulated in the cerebral cortex (sham, $1.3 \pm 0.3$ per $100 \mu \mathrm{m}^{2}$; 4 -week mCCAO, 6.5 \pm 0.3 per $100 \mu \mathrm{m}^{2} ; \mathrm{p}=0.004, F=1.6 ; 8$-week mCCAO, 5.2 \pm 0.7 per $100 \mu \mathrm{m}^{2} ; \mathrm{p}=0.005, F=1.9$; Fig. 7 ), indicating that $\mathrm{CCH}$ caused by mCCAO triggered angiogenesis and microcirculation changes in the cerebral cortex.

\section{Summary of Changes at Extended Time Point}

No significant differences were observed between 4-week and 8-week mCCAO rats regarding neuronal cell death, glial activation, and angiogenesis (data not shown). After 8 weeks of mCCAO, despite reversal of the WM injury, cognitive dysfunction remained and may be deteriorated in part. No significant differences were noted between right and left sides of rat brains at 4 and 8 weeks of mCCAO (data not shown).

\section{Discussion}

Our modified $\mathrm{CCH}$ model was quick and straightforward and had a low mortality rate. The rCBF declined moderately before gradually recovering for up to 4 weeks with the development of collateral circulation from the vertebral and basilar arteries. Our mCCAO caused WM injury and hippocampal cell death with glial activation and angiogenesis. These results, in combination with cognitive impairment, appear to be very similar to the changes seen in vascular dementia as well as the pathophysiology of moyamoya disease, a cerebrovascular chronic occlusive disease with microcirculation changes that were partly elucidated in our model by the activation of microvessels and rCBF changes in line with previous studies..$^{14,34}$ Reportedly, the most prevalent treatment for moyamoya disease, indirect bypass (e.g., encephalomyosynangiosis), could be improved through combination with gene or stem cell therapy ${ }^{8,11}$ As our mCCAO model can be easily combined with indirect bypass or cell therapies, it can facilitate studying the pathophysiology of vascular dementia and moyamoya disease.

In our model, 2 types of glial cells (astrocytes and microglia) were activated at 4 and 8 weeks. Of note, T2weighted MRI after mCCAO demonstrated no areas of infarction, as have been reported to occur after BCCAO because of the sudden decrease in the rCBF., $5,15,22,26,28$ Perhaps the unilateral CCA stenosis of our model could maintain minimal CBF in the early stage, although it might be thrombosed in the chronic phase, possibly facilitating the development of the collateral circulation from the vertebral and basilar arteries, and inducing a moderate global hypoperfusion state leading to equivalent changes in both sides of the brain, as evident on the histological evaluation, unlike previously reported unilateral CCA occlusion. ${ }^{34}$

The highlights of our model are its low mortality rate and the ease with which the procedure may be performed. The survival rate of the standard BCCAO model has been reported to be $56.5 \%-64.6 \%$ in Sprague-Dawley rats. $7,10,12,15,35$ Staged BCCAO has been associated with 
A
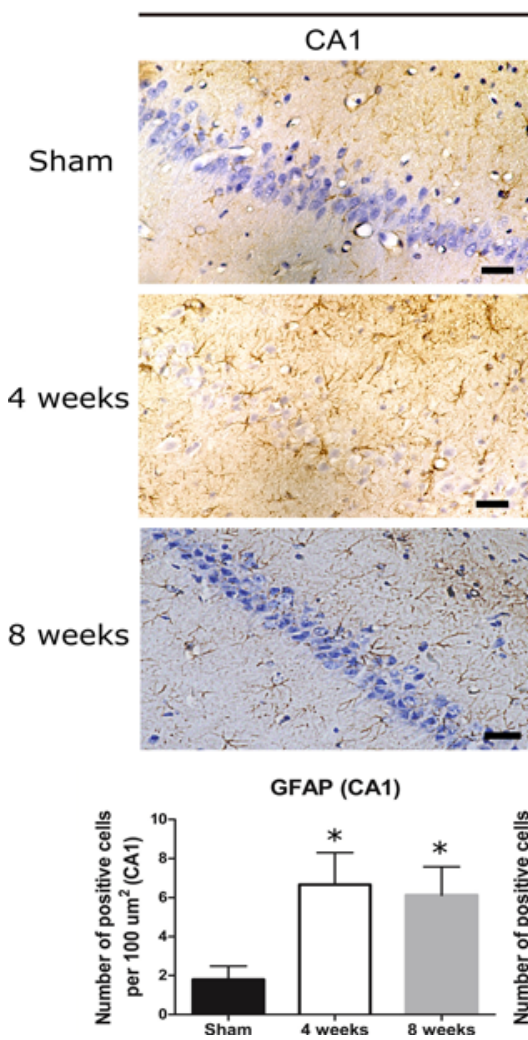

GFAP
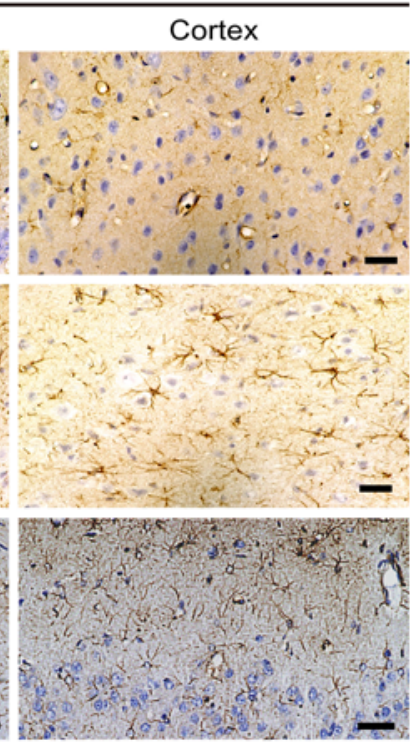

GFAP (cortex)

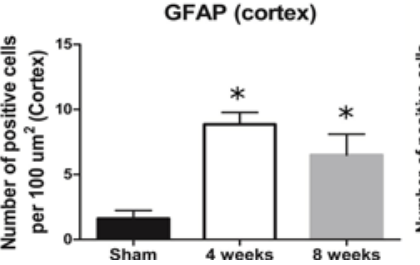

B

Iba-1
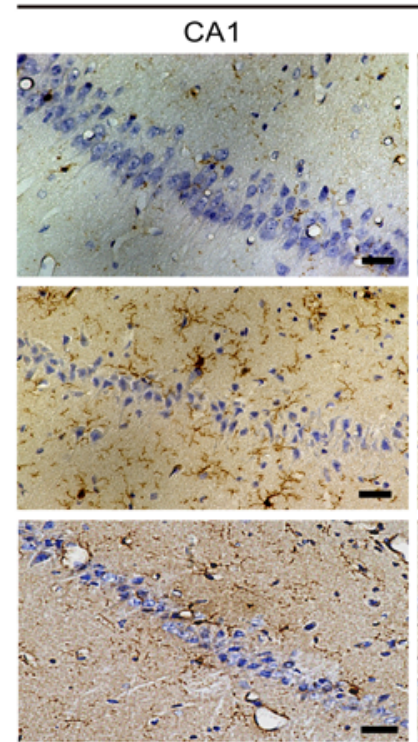

Iba-1 (CA1)

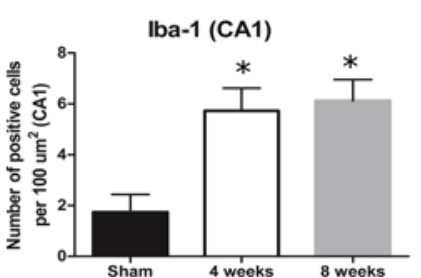

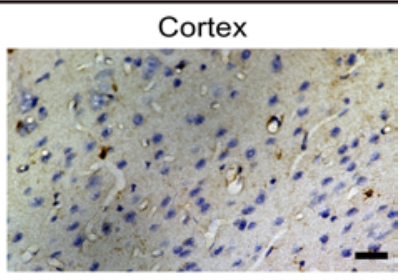
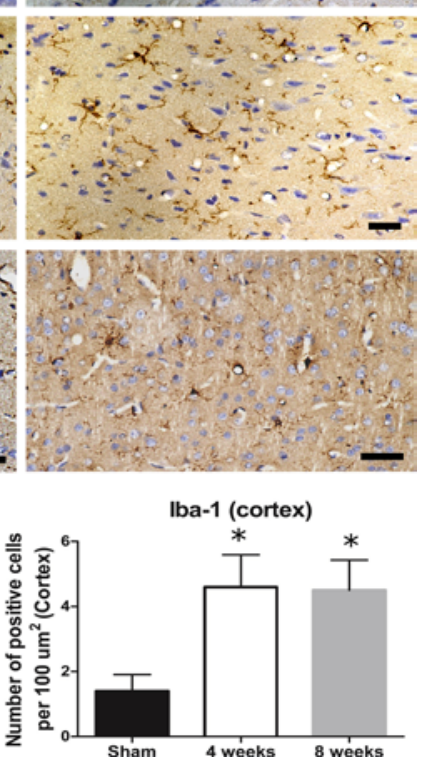

FIG. 6. Inflammatory changes in the parietal cortex and the hippocampal CA1 subregion. The number of cells positive for GFAP $\left(A,{ }^{*} p=0.001\right)$ and lba-1 $\left(B,{ }^{*} p=0.002\right)$ was significantly increased in the bilateral parietal cortex and hippocampal CA1 subregion 4 and 8 weeks after mCCAO compared to findings in sham-operated rats. Photomicrographs, original magnification 200x; bar = $100 \mathrm{um}$. The graph shows the means of 15 rats for the sham group, 21 rats for the mCCAO group at 4 weeks, and 8 rats for the mCCAO at 8 weeks; error bars indicate standard deviations.

a somewhat reduced but still high mortality rate.,12,15,35 Although the use of microcoils to develop stenosis in mice has been reported to yield more stable results than $\mathrm{CCAO}, 7,12,35$ the risk of causing endothelial injury and the cost of microcoils warrant consideration. In this study, we assessed CBF by LSF using the skull-thinning technique in contrast to previous use of a completely open skull win- dow. ${ }^{12}$ Our technique offers an easier procedure for serially following LSF and avoiding injury to the dura mater.

The compensation of CBF showed a strong relationship to the development of collateral circulation. In our model, the CBF demonstrated a moderate progressive reduction over the 1st week, plateaued at the 2nd week, and then started recovering from the 3 rd to 4 th week; however,

\section{CD34 (cortex)}
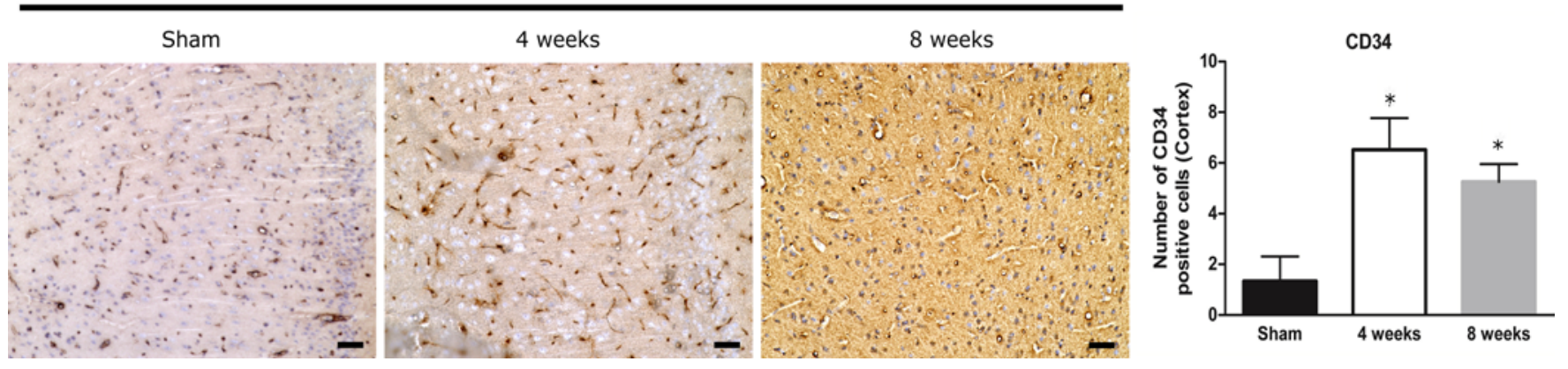

FIG. 7. Immunohistochemistry of CD34. The number of CD34-positive microvessels was significantly increased in the parietal cortex 4 and 8 weeks after $m C C A O$ compared to findings in the sham group ( $\left.{ }^{*} p=0.004\right)$. Photomicrographs, original magnification $200 x ;$ bar $=100 \mu \mathrm{m}$. The graph shows the means of 15 rats for the sham group, 21 rats for the mCCAO group at 4 weeks, and 8 rats for the mCCAO group at 8 weeks, 3 sections per animal averaged from the same location of the parietal cortex; error bars indicate standard deviations. 
this recovery did not affect the histological and behavioral sequelae of $\mathrm{CCH}$ as assessed at 4 and 8 weeks after the surgery. Possible mechanisms for recovering the $\mathrm{CBF}$ in $\mathrm{CCH}$ could be associated with the biomedical regulation of the $\mathrm{CBF}$, recruitment of nonperfused capillaries, angiogenesis, and dilation of vertebral and basilar arteries. ${ }^{7,10}$ In this study, we observed 2 compensation mechanisms: 1) at the macrovascular level, the dilation of vertebral and basilar arteries was observed by 3D-TOF MRA, which showed increased tortuosity and diameter of both VAs; and 2) at the microvascular level, angiogenesis (demonstrated CD34-positive cells on immunohistochemical analysis) was observed at 4 and 8 weeks after the surgery. In line with previous studies, capillary densities in both frontal and parietal cortices increased to approximately 1.3 times the control level at 28 days after the surgery. ${ }^{10,13}$

At 4 and 8 weeks after the mCCAO procedure, neuronal death in the cerebral cortex and hippocampus and the associated cognitive impairment were evident in this study. Several studies have demonstrated degeneration of different cell types in $\mathrm{CCH} ;, 4,7,26,28$ these findings were further supported in this study, which showed the percentage of damaged neurons in the CA1 subregion to be around $30 \%$ at 4 weeks after mCCAO and $23 \%$ at 8 weeks after mCCAO. The cognitive impairment assessment by BCM in our model further validated that $\mathrm{CCH}$ induces impairment in spatial learning and memory. ${ }^{6,7,12,17,20,29,31,32,35}$

In our study, WM injury was evident 1 week after mCCAO and might contribute to the development of cognitive impairment of the $\mathrm{CCH}$ as previously described. ${ }^{12,15}$, 21,24,25,29 In addition, resolution of the WM injury may start after 4 weeks and become more evident at 8 weeks. In the central nervous system, such resolution is rare; thus, our model could be a tool to investigate the molecular pathology of and develop new treatments for WM lesions.

This study has some limitations. First, we did not completely establish irreversibility of the cognitive function and the detailed associated behavioral functions or related changes at cellular and subcellular levels. Second, although we would have liked to have been able to subject all animals in each group to all experiments, technical difficulties and equipment availability for MRI and the BCM prevented this. Finally, LSF only measured the cortical CBF, and we could not evaluate deep brain microcirculation and CBF changes. The precise evaluation of microcirculatory changes other than angiogenesis requires more advanced imaging tools. Overall, further mechanistic evaluations and extensive treatment trials with better equipment are warranted in future studies.

\section{Conclusions}

This refined $\mathrm{CCH}$ model using mCCAO in rats is straightforward and is associated with a low mortality rate. It has potential for the investigation of vascular dementia, moyamoya disease, and other $\mathrm{CCH}$ pathophysiology and treatment options, as well as studying angiogenesis and neuroinflammation.

\section{Acknowledgments}

We thank Hiroi Nonaka for the technical assistance for MRI and Maki Sugawara for the assistance for the preparation of frozen blocks of rat brains and for cryosection. We would also like to thank Enago for the English language review.

\section{References}

1. Cechetti F, Pagnussat AS, Worm PV, Elsner VR, Ben J, da Costa MS, et al: Chronic brain hypoperfusion causes early glial activation and neuronal death, and subsequent long-term memory impairment. Brain Res Bull 87:109-116, 2012

2. Choi DH, Lee KH, Lee J: Effect of exercise-induced neurogenesis on cognitive function deficit in a rat model of vascular dementia. Mol Med Rep 13:2981-2990, 2016

3. Dias Fiuza Ferreira E, Valério Romanini C, Cypriano PE, Weffort de Oliveira RM, Milani H: Sildenafil provides sustained neuroprotection in the absence of learning recovery following the 4-vessel occlusion/internal carotid artery model of chronic cerebral hypoperfusion in middle-aged rats. Brain Res Bull 90:58-65, 2013

4. Du SQ, Wang XR, Xiao LY, Tu JF, Zhu W, He T, et al: Molecular mechanisms of vascular dementia: What can be learned from animal models of chronic cerebral hypoperfusion? Mol Neurobiol 54:3670-3682, 2017

5. Farkas E, Luiten PG, Bari F: Permanent, bilateral common carotid artery occlusion in the rat: a model for chronic cerebral hypoperfusion-related neurodegenerative diseases. Brain Res Brain Res Rev 54:162-180, 2007

6. Hai J, Wan JF, Lin Q, Wang F, Zhang L, Li H, et al: Cognitive dysfunction induced by chronic cerebral hypoperfusion in a rat model associated with arteriovenous malformations. Brain Res 1301:80-88, 2009

7. Hattori Y, Enmi J, Kitamura A, Yamamoto Y, Saito S, Takahashi Y, et al: A novel mouse model of subcortical infarcts with dementia. J Neurosci 35:3915-3928, 2015

8. Hiramatsu M, Hishikawa T, Tokunaga K, Kidoya H, Nishihiro S, Haruma J, et al: Combined gene therapy with vascular endothelial growth factor plus apelin in a chronic cerebral hypoperfusion model in rats. J Neurosurg 127:679-686, 2017

9. Huang Y, Fan S, Li J, Wang YL: Bilateral common carotid artery occlusion in the rat as a model of retinal ischaemia. Neuroophthalmology 38:180-188, 2014

10. Jing Z, Shi C, Zhu L, Xiang Y, Chen P, Xiong Z, et al: Chronic cerebral hypoperfusion induces vascular plasticity and hemodynamics but also neuronal degeneration and cognitive impairment. J Cereb Blood Flow Metab 35:12491259,2015

11. Kim HS, Lee HJ, Yeu IS, Yi JS, Yang JH, Lee IW: The neovascularization effect of bone marrow stromal cells in temporal muscle after encephalomyosynangiosis in chronic cerebral ischemic rats. J Korean Neurosurg Soc 44:249-255, 2008

12. Kitamura A, Saito S, Maki T, Oishi N, Ayaki T, Hattori Y, et al: Gradual cerebral hypoperfusion in spontaneously hypertensive rats induces slowly evolving white matter abnormalities and impairs working memory. J Cereb Blood Flow Metab 36:1592-1602, 2016

13. Kusaka N, Sugiu K, Tokunaga K, Katsumata A, Nishida A, Namba K, et al: Enhanced brain angiogenesis in chronic cerebral hypoperfusion after administration of plasmid human vascular endothelial growth factor in combination with indirect vasoreconstructive surgery. J Neurosurg 103:882-890, 2005

14. Leardini-Tristão M, Borges JP, Freitas F, Rangel R, Daliry A, Tibiriçá E, et al: The impact of early aerobic exercise on brain microvascular alterations induced by cerebral hypoperfusion. Brain Res 1657:43-51, 2017

15. Li N, Gu Z, Li Y, Fu X, Wang J, Bai H: A modified bilateral carotid artery stenosis procedure to develop a chronic cere- 
bral hypoperfusion rat model with an increased survival rate. J Neurosci Methods 255:115-121, 2015

16. Long Q, Hei Y, Luo Q, Tian Y, Yang J, Li J, et al: BMSCs transplantation improves cognitive impairment via up-regulation of hippocampal GABAergic system in a rat model of chronic cerebral hypoperfusion. Neuroscience 311:464-473, 2015

17. Lu Y, Li C, Zhou M, Luo P, Huang P, Tan J, et al: Clonidine ameliorates cognitive impairment induced by chronic cerebral hypoperfusion via up-regulation of the $\mathrm{GABA}_{\mathrm{B}} \mathrm{R} 1$ and GAD67 in hippocampal CA1 in rats. Pharmacol Biochem Behav 132:96-102, 2015

18. Luo P, Lu Y, Li C, Zhou M, Chen C, Lu Q, et al: Long-lasting spatial learning and memory impairments caused by chronic cerebral hypoperfusion associate with a dynamic change of HCN1/HCN2 expression in hippocampal CA1 region. Neurobiol Learn Mem 123:72-83, 2015

19. Matin N, Fisher C, Jackson WF, Dorrance AM: Bilateral common carotid artery stenosis in normotensive rats impairs endothelium-dependent dilation of parenchymal arterioles. Am J Physiol Heart Circ Physiol 310:H1321-H1329, 2016

20. Miki K, Ishibashi S, Sun L, Xu H, Ohashi W, Kuroiwa T, et al: Intensity of chronic cerebral hypoperfusion determines white/gray matter injury and cognitive/motor dysfunction in mice. J Neurosci Res 87:1270-1281, 2009

21. Nakaji K, Ihara M, Takahashi C, Itohara S, Noda M, Takahashi R, et al: Matrix metalloproteinase-2 plays a critical role in the pathogenesis of white matter lesions after chronic cerebral hypoperfusion in rodents. Stroke 37:2816-2823, 2006

22. Neto CJ, Paganelli RA, Benetoli A, Lima KC, Milani H: Permanent, 3-stage, 4-vessel occlusion as a model of chronic and progressive brain hypoperfusion in rats: a neurohistological and behavioral analysis. Behav Brain Res 160:312-322, 2005

23. Nishino A, Tajima Y, Takuwa H, Masamoto K, Taniguchi J, Wakizaka H, et al: Long-term effects of cerebral hypoperfusion on neural density and function using misery perfusion animal model. Sci Rep 6:25072, 2016

24. Sarti C, Pantoni L, Bartolini L, Inzitari D: Cognitive impairment and chronic cerebral hypoperfusion: what can be learned from experimental models. J Neurol Sci 203204:263-266, 2002

25. Shibata M, Ohtani R, Ihara M, Tomimoto H: White matter lesions and glial activation in a novel mouse model of chronic cerebral hypoperfusion. Stroke 35:2598-2603, 2004

26. Soria G, Tudela R, Márquez-Martín A, Camón L, Batalle $\mathrm{D}$, Muñoz-Moreno E, et al: The ins and outs of the BCCAo model for chronic hypoperfusion: a multimodal and longitudinal MRI approach. PLoS One 8:e74631, 2013

27. Ueno Y, Koike M, Shimada Y, Shimura H, Hira K, Tanaka $\mathrm{R}$, et al: L-carnitine enhances axonal plasticity and improves white-matter lesions after chronic hypoperfusion in rat brain. J Cereb Blood Flow Metab 35:382-391, 2015

28. Venkat P, Chopp M, Chen J: Models and mechanisms of vascular dementia. Exp Neurol 272:97-108, 2015

29. Wakita H, Tomimoto H, Akiguchi I, Matsuo A, Lin JX, Ihara M, et al: Axonal damage and demyelination in the white mat- ter after chronic cerebral hypoperfusion in the rat. Brain Res 924:63-70, 2002

30. Wang J, Fu X, Jiang C, Yu L, Wang M, Han W, et al: Bone marrow mononuclear cell transplantation promotes therapeutic angiogenesis via upregulation of the VEGF-VEGFR2 signaling pathway in a rat model of vascular dementia. Behav Brain Res 265:171-180, 2014

31. Wang J, Fu X, Yu L, Li N, Wang M, Liu X, et al: Preconditioning with VEGF enhances angiogenic and neuroprotective effects of bone marrow mononuclear cell transplantation in a rat model of chronic cerebral hypoperfusion. Mol Neurobiol 53:6057-6068, 2016

32. Wang Z, Fan J, Wang J, Li Y, Duan D, Du G, et al: Chronic cerebral hypoperfusion induces long-lasting cognitive deficits accompanied by long-term hippocampal silent synapses increase in rats. Behav Brain Res 301:243-252, 2016

33. Yata K, Tomimoto H: Chronic cerebral hypoperfusion and dementia. Neurol Clin Neurosci 2:129-134, 2014

34. Yoshizaki K, Adachi K, Kataoka S, Watanabe A, Tabira T, Takahashi K, et al: Chronic cerebral hypoperfusion induced by right unilateral common carotid artery occlusion causes delayed white matter lesions and cognitive impairment in adult mice. Exp Neurol 210:585-591, 2008

35. Zhou Z, Zhang Y, Zhu C, Sui J, Wu G, Meng Z, et al: Cognitive functions of carotid artery stenosis in the aged rat. Neuroscience 219:137-144, 2012

\section{Disclosures}

This work was partially supported by JSPS KAKENHI Grant Number $17 \mathrm{H} 01583$ and the Project for Japan Translational and Clinical Research Core Centers (No. 17lm0203026h0001) from the Japan Agency for Medical Research and Development, AMED. The authors report no conflict of interest concerning the materials or methods used in this study or the findings specified in this paper.

\section{Author Contributions}

Conception and design: Niizuma, Mansour. Acquisition of data: Mansour. Analysis and interpretation of data: Niizuma, Mansour, Sumiyoshi, Ryoke. Drafting the article: Mansour. Critically revising the article: Niizuma, Rashad, Sumiyoshi, Ryoke, H Endo, T Endo, Sato, Kawashima, Tominaga. Reviewed submitted version of manuscript: Niizuma, Rashad, Sumiyoshi, Ryoke, H Endo, T Endo, Sato, Kawashima, Tominaga. Statistical analysis: Mansour, Ryoke. Administrative/technical/material support: Niizuma, Sumiyoshi, Kawashima, Tominaga. Study supervision: Niizuma, Tominaga.

\section{Correspondence}

Kuniyasu Niizuma: Tohoku University Graduate School of Biomedical Engineering, Sendai, Japan.niizuma@nsg.med. tohoku.ac.jp. 